\title{
Extended-Spectrum Beta-Lactamases Producing Microorganisms isolated from UTI Patients: an Alarm
}

\author{
Arti Agrawal $^{1 *}$, Namita Srivastava', ${ }^{1}$ ikas Kumar ${ }^{1}$, Neha Bhati ${ }^{2}$, \\ Rashmi $^{2}$ and Ankur Goyal ${ }^{1}$ \\ ${ }^{1}$ Department of Microbiology, Sarojini Naidu Medical College, Agra (Uttar Pradesh), India \\ ${ }^{2}$ Department of Microbiology, Chhatrapati Shahu Ji Maharaj University, \\ Kanpur (Uttar Pradesh), India \\ *Corresponding author
}

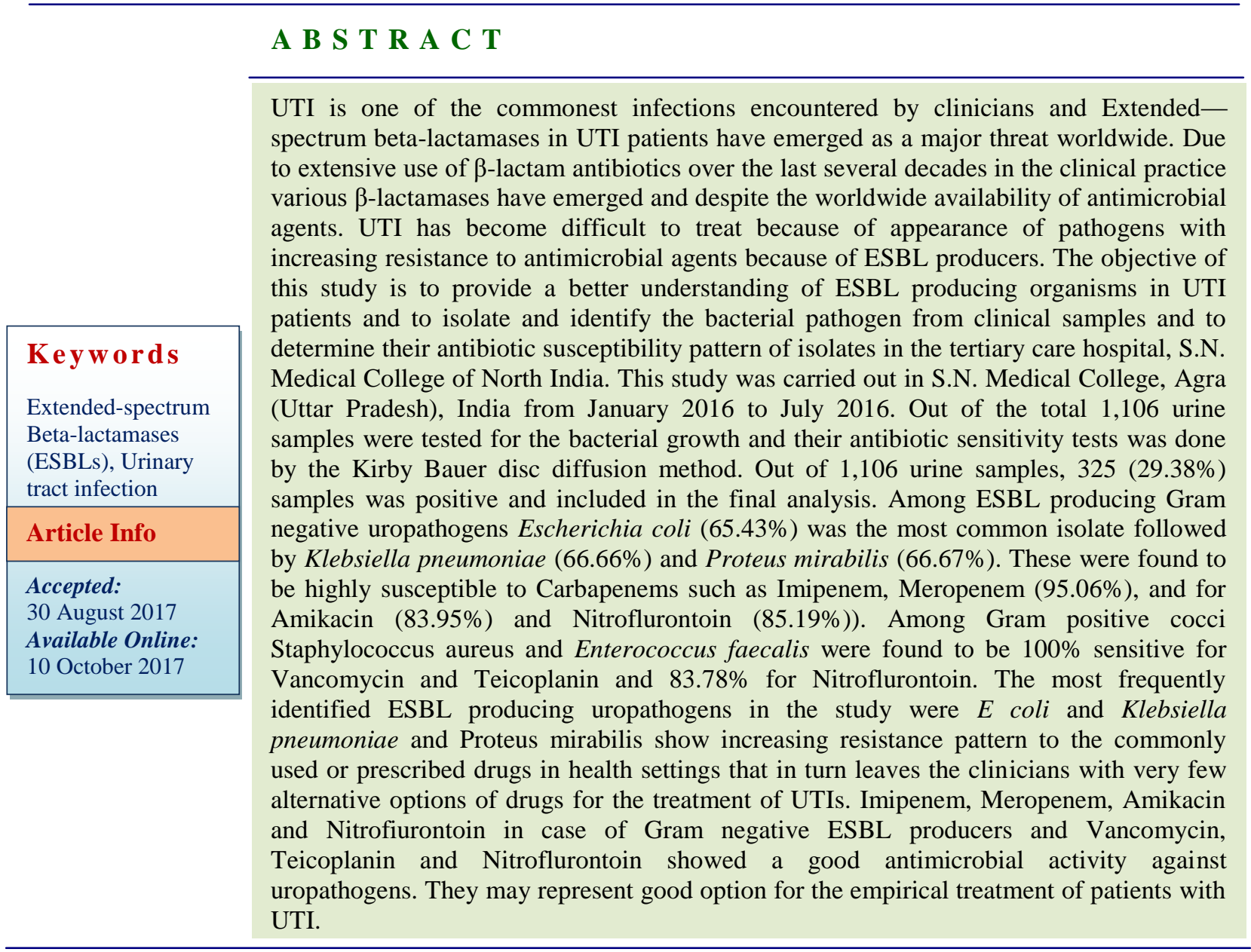

\section{Introduction}

Globally, Extended-spectrum of $\beta$ - lactamases ESBLs are considered to be most problematic, particularly in hospitalized patients and those undergoing long term treatment with limited 
options. Infections with ESBL producing organisms have been associated with poor outcomes. Extended-spectrum $\beta$ - lactamases are the enzymes mainly produced by Gram negative bacilli that have the ability to destroy or break down one or more extended spectrum antibiotics belonging to the cephalosporins (beta lactam antibiotics like ceftazidime, ceftriazone, cefotoxime, and cefepime etc), monobactam aztreonam and penicillines, containing an oxyimino group and render them ineffective (1). It is one of the ways in which bacteria develop resistance. With increasing multidrug resistance in uropathogens is an important and emerging public health problem. The prevalence of antimicrobial resistance among uropathogens have been increasing worldwide due to irrational use of antibiotics in practise $(2,3)$. ESBL infections have occurred in people who are already very sick and in elderly people and also those patients who have been taking antibiotics or previously hospitalized are mainly affected (4). As a result, infections caused by these bacteria can be difficult to treat and infections caused by these bacteria are become more common in both community and health care settings. They are usually plasmid mediated $\beta$ - lactamases that can be exchanged between bacteria and most commonly found in Gram negative bacteria especially in the member of Enterobacteriaceae such as Escherichia coli, Klebsiella pneumoniae and Proteus mirabilis and Pseudomonas aeruginosa $(1,5)$. The first plasmid mediated $\beta$-lactamase in Gram negative bacteria was discovered in Greece in the 1960s and it was named TEM (1). The first derivation of TEM-1 is TEM-2 with a single replacement of amino acid (6). Another common prevalent type of beta- lactamases is SHV-1 that was reported initially in Klebsiella pneumoniae. It is estimated that $\mathrm{SHV}-1$ is responsible for plasmid mediated ampicillin resistance in bacteria that harbours it. The replacement of single amino acids causes the change in enzyme structures and affects its activities (1). A substitution is more common among TEM, SHV and OXA enzymes in defined amino acids positions. The combination of altered amino acids produce variety of phenotypes of betalactamases enzymes with varying ability to breakdown $3^{\text {rd }}$ generation cephalosporins and increase their level of resistance to betalactamases inhibitors (7). They can be inhibited by the action of $\beta$ - lactamases inhibitors such as clavulanic acid, salbactams and tazobactams (1). This study is important for clinicians in order to facilitate the effective treatment and management of patient with symptoms of urinary tract infections.

\section{Materials and Methods}

This study was carried out in a tertiary care centre of North India, in the Department of Microbiology, S.N. Medical College, Agra (Uttar Pradesh), India, during the period of January 2016 to July 2016. The urine samples were collected from different wards of OPD (outpatient department), NICU, PICU, ED pedia, Gynae OPD.

The total of 1,106 urine samples were analysed and patients were advised to collect the clean catch midstream urine into a $50 \mathrm{ml}$ calibrated sterile screw capped container. The specimens were labelled, transported to the laboratory as early as possible. Isolation and identification of bacterial pathogens was done by the microscopy and culture methods. Microscopy was done by Gram's staining and culture method was done with a loopful of the well mixed urine samples were inoculated onto Cystine-Lactose-Electrolyte-Deficient (CLED) agar medium to determine the colony forming unit (CFU). After incubated at $37^{\circ} \mathrm{C}$ aerobically for 24-48 hours and then examined for positive bacterial growth. A specimen was considered positive for UTI 
infection, if an organism was cultured at a concentration of $\geq 10^{5} \mathrm{cfu} / \mathrm{ml}$ and less than $10^{2} \mathrm{cfu} / \mathrm{ml}$ was interpreted as negative (8). Bacterial isolates were identified generally by performing conventional biochemical tests.

\section{Detection of ESBL producers}

This test was done by using double disc synergy test (DDST) on Muller Hinton agar medium with cephalosporin alone and cephalosporin with combination of $\beta$ - lactam inhibitors like clavulanic acid, tazobactam and sulbactams. The positive and negative control strains used in this test were Klebsiella pneumoniae ATCC 700603 and E. coli ATCC 25922, respectively.

\section{Antimicrobial Susceptibility Testing}

All isolates were tested for antimicrobial susceptibility testing by the standard Kirby Bauer's disc diffusion method on Muller Hinton Agar medium. After incubation of 24 hours the inhibition zones were measured and interpreted according to clinical and lab standards institute (CLSI) guidelines (9) using the following standard antibiotic discs for the isolates i.e, Ciprofloxacin $(05 \mu \mathrm{g})$,

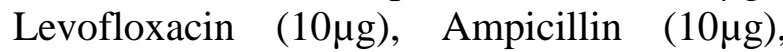
Ampicillin-Sulbactam (10/10 $\mu$ g), Ceftazidime (30 $\mu \mathrm{g}), \quad$ Ciftriaxone/Salbactam $\quad(30 \mu \mathrm{g})$, Cefepime $(30 \mu \mathrm{g})$, Gentamicin $(10 \mu \mathrm{g})$, Doxycycline $(30 \mu \mathrm{g})$, Tetracycline $(30 \mu \mathrm{g})$, Teicoplanin $(30 \mu \mathrm{g})$, Nitrofurantoin $(300 \mu \mathrm{g})$, Amikacin $\quad(30 \mu \mathrm{g}), \quad$ Cefaclor $(30 \mu \mathrm{g})$, Cotrimoxazole $(1.25 / 23.75 \mu \mathrm{g})$, High Level Gentamycin $(120 \mu \mathrm{g})$, Vancomycin $(30 \mu \mathrm{g})$, Piperacillin / Tazobactam (100/10 $\mu \mathrm{g})$, Polymyxin-B (300units), Aztreonam $(30 \mu \mathrm{g})$, Colistin $\quad(10 \mu \mathrm{g}), \quad$ Imipenam $(10 \mu \mathrm{g})$, Meropenem $(10 \mu \mathrm{g})$ etc.

\section{Results and Discussion}

Out of the total 1,106 specimens, 325 $(29.38 \%)$ were positive with identified pathogens and are subjected to antimicrobial susceptibility testing. Among 325, $173(53.23 \%)$ were female patients and 152 $(46.77 \%)$ were males. The most common isolate in this study have been the Gram negative bacilli.

$E$ coli was the most frequently identified Gram negative bacteria accounted for 162 $(49.84 \%)$ followed by other uropathogens like Enterococcus faecalis (11.38\%), Klebsiella pneumoniae (6.46\%), Proteus mirabilis (2.76\%), Staphylococcus aureus/sp (2.46\%), Pseudomonas aeruginosa (2.15\%) among the major isolates. (Table No. 1)

\section{ESBL producers}

Among ESBL producers the predominant isolate was E coli 106(65. 43\%) followed by Klebsiella pneumoniae 14(66.66\%) and Proteus mirabilis 6(66.67\%). (Figure-1)

\section{Antibiotic susceptibility test}

Among the tested antibiotics the highest susceptibility for Gram negative bacteria was shown by carbepenems such as Imipenem, Meropenem, Nitrofurantoin and Amikacin. In our study the resistance rate of isolated uropathogens to fluoroquinolones, second and third generation cephalosporins was high ranging from (70\% to $90 \%)$.

In ESBL producer, $E$ coli was the predominant isolate were susceptible to Imipenem (95.06\%), Meropenem (95.06\%), Nitrofurantoin $(85.19 \%)$ Amikacin $(83.95 \%)$. Cefepime and Ceftazidime (74.07\%) resistance indicates ESBL production. (Table no.3). Ps. aeruginosa were found $100 \%$ susceptible for Vancomycin and Teicoplanin and $98 \%$ for Imipenem and $71.43 \%$ for Ceftazidime and Aztreonam. Similarly $E$. faecalis and S. aureus were showed $100 \%$ susceptibility for Vancomycin and Teicoplanin and $83.78 \%$ for Nitroflurantoin. 
Table.1 Distribution of uropathogens among both gender

\begin{tabular}{|l|l|l|l|l|}
\hline S. no & Isolated Uropathogen & $\begin{array}{l}\text { Female (\%) } \\
\mathrm{n}-173(53.23)\end{array}$ & $\begin{array}{l}\text { Male (\%) } \\
\mathrm{n}-152(46.77)\end{array}$ & $\begin{array}{l}\text { Total no of } \\
\text { patients- n-325 }\end{array}$ \\
\hline 1 & Escherichia coli & $84(51.85)$ & $78(48.15)$ & $162(49.84)$ \\
\hline 2 & Enterococcus faecalis & $18(48.65)$ & $19(51.35)$ & $37(11.38)$ \\
\hline 3 & Klebsiella pneumoniae & $13(61.90)$ & $8(38.09)$ & $21(6.46)$ \\
\hline 4 & Proteus mirabilis & $3(33.33)$ & $6(66.66)$ & $9(2.76)$ \\
\hline 5 & Staphylococcus aureus & $6(75)$ & $2(25)$ & $8(2.46)$ \\
\hline 6 & Pseudomonas aeruginosa & $1(14.29)$ & $6(85.71)$ & $7(2.15)$ \\
\hline 7 & Candida sp. & $48(59.26)$ & $33(40.74)$ & $81(24.92)$ \\
\hline
\end{tabular}

$\mathrm{n}=$ Total no. of patients

Table.2 Susceptibility rates (\%) for isolated Gram negative uropathogens

\begin{tabular}{|l|l|l|l|l|}
\hline & \multicolumn{5}{|c|}{ Isolated Uropathogen } \\
\hline A/B & $\begin{array}{l}\boldsymbol{E} \text { coli } \\
(\mathbf{n}-\mathbf{1 6 2})\end{array}$ & $\begin{array}{l}\text { K. pneumoniae } \\
(\mathbf{n - 2 1})\end{array}$ & $\begin{array}{l}\text { Proteus mirabilis } \\
(\mathbf{n}-\mathbf{9})\end{array}$ & $\begin{array}{l}\text { Ps. aeruginosa } \\
(\mathbf{n}-\mathbf{7})\end{array}$ \\
\hline & Sensitive (\%) & Sensitive (\%) & Sensitive (\%) & Sensitive (\%) \\
\hline Imipenem & 95.06 & 95.24 & 88.89 & 85.72 \\
\hline Meropenem & 95.06 & 90.48 & 88.89 & 86.41 \\
\hline Nitrofurantoin & 85.19 & 52.38 & 00.00 & 00.00 \\
\hline Amikacin & 83.95 & 61.90 & 77.78 & 71.42 \\
\hline Doxycycline & 39.51 & 23.80 & 44.45 & 42.85 \\
\hline Tetracycline & 38.27 & 23.80 & 44.45 & 42.85 \\
\hline Gentamicin & 40.74 & 42.86 & 66.67 & 14.29 \\
\hline Cefepime & 25.93 & 28.57 & 33.33 & 28.57 \\
\hline Ceftazidime & 24.69 & 23.80 & 44.44 & 71.43 \\
\hline Ceftriaxone/sulbactum & 66.04 & 66.66 & 66.67 & 57.14 \\
\hline Levofloxacin & 12.35 & 19.05 & 22.23 & 28.57 \\
\hline Ciprofloxacin & 11.73 & 28.57 & 22.23 & 28.57 \\
\hline Moxifloxacin & 12.35 & 23.80 & 33.34 & 14.28 \\
\hline Ampicillin & 7.41 & 00.00 & 11.12 & 14.28 \\
\hline Amoxyclavulanic acid & 30.86 & 23.80 & 22.23 & - \\
\hline Cotrimoxazole & - & 9.52 & 77.78 & - \\
\hline Polymycin-B & - & - & - & 100 \\
\hline Colistin & - & - & - & 71.43 \\
\hline Azetreonam & - & - & - & 42.86 \\
\hline Piperacillin/tazobactum & - & - & - & \\
\hline
\end{tabular}


Table.3 Susceptibility rates (\%) for Gram positive uropathogen

\begin{tabular}{|l|l|l|}
\hline Antibiotic & \multicolumn{2}{|c|}{ Isolated Uropathogen } \\
\hline & E. faecalis (n-37) & S. aureus (n-8) \\
\hline & Sensitive (\%) & Sensitive (\%) \\
\hline Vancomycin & 100 & 100 \\
\hline Teicoplanin & 100 & 100 \\
\hline Nitrofurantion & 83.78 & - \\
\hline Doxycycline & 45.95 & 62.5 \\
\hline Tetracycline & 51.35 & 62.5 \\
\hline Ampicillin & 13.51 & - \\
\hline Ampicillin/salbactum & 13.51 & 37.5 \\
\hline Amoxyclavunic acid & 18.92 & - \\
\hline Levofloxacin & 16.22 & 12.5 \\
\hline Cotrimoxazole & 13.51 & 25 \\
\hline High-level gentamycin & 37.84 & - \\
\hline Cefaclor & - & 25 \\
\hline Piperacillin/tazobactum & - & 37.5 \\
\hline Cefepime & - & 25 \\
\hline Penicillin-G & 0 & 0 \\
\hline Clindamycin & - & 62.5 \\
\hline
\end{tabular}

Fig.1 Distribution of ESBL producers of isolated Uropathogen

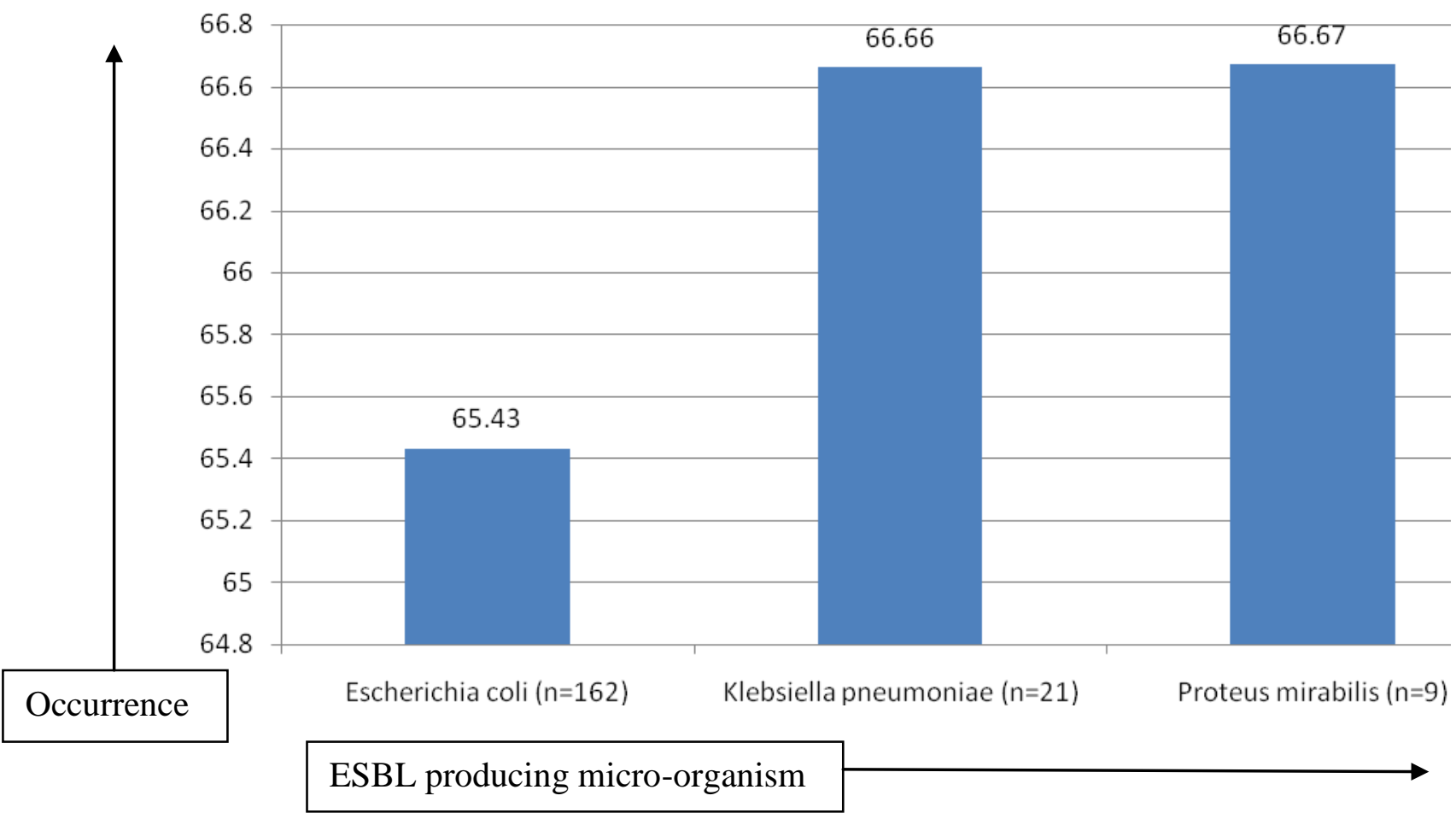


Urinary tract infections are the common clinical condition worldwide. A variety of enteropathogenic bacteria are known to cause UTI. In our study we described the relationship between sex, isolated uropathogens and their antibiotic susceptibility. This study showed a higher prevalence of UTI in females $(53.23 \%)$ than in males (46.77\%) which is analogous with those of other findings revealing that the frequency of UTI is higher in females as compared to males $(10,11$,$) . Our study$ showed that $E$ coli $(49.84 \%)$ was the commonest Gram negative uropathogen identified in both gender as described previously $(12,13)$. Other Gram negative uropathogens known to cause UTI including Klebsiella pneumoniae, Pseudomonas aeruginosa, Proteus mirabilis and Gram positive Staphylococcus aureus and Enterococcus faecalis etc., these also correspond to the data obtained by other groups $(14,15)$. Second common isolated uropathogen in our study was Enterococcus faecalis showed higher prevalence in men than in women, similar to other findings (16) and differ from other studies as explained Klebsiella pneumoniae was second lead uropathogen (17). The similarities and differences are based on the type and distribution of uropathogens and they may result from different environmental conditions and host factors. On the basis of our study the resistance rate of isolated uropathogens mainly in E coli and Klebsiella pneumoniae for flouroquinolones includes ciprofloxacin, Levofloxacin, Moxifloxacin etc and to second and third generation cephalosporines was showed higher resistance. The resistance rate of upto 70-90\% against flouroquinolones and upto $80-90 \%$ against cephalosporines. This is similar to the rate of antibiotics resistance previously reported studies $(18,19)$. The clinical trials conducted worldwide have reported that these antibiotics are the most common used ones because of their easier administration and cheaper availability. And also a hisgher proportion of uropathogens were resistance to ampicillin, Cotrimaxazole and amoxicillin. The emergence of resistance for flouroquinolones is multifactorial due to an important mechanism of antibiotic resistance among uropathogens is through ESBL production. Among the Gram negative uropathogens, the emergence of resistance to extended spectrum cephalosporins has been a major concern (19). Mainly Gram negative bacteria are the common host of ESBL producers such as $E$ coli, Klebsiella pneumoniae and Proteus mirabilis of these $E$ coli is the one of main ESBL producing pathogen. Production of ESBL is plasmid mediated and this plasmid carries multiple drug resistance (MDR) genes against aminoglycosides, quinolones and sulfamethoxazole at the same time (20). MDR increases the number of difficulties to the clinical treatments due to limited therapeutic options $(21,22)$. It has been reported that Amikacin, Imipenem. Meropenem and Nitrofurantoin are the most affective antibiotics (in case of ESBL producers) against E coli, Klebsiella pneumoniae and Proteus mirabilis (23). Our study further supported by another study where the susceptibility rate of $E$ coli and Klebsiella pneumoniae to Amikacin remained 80-90\% (24). Carbepenem such as Meropenem, Imipenem were found to be most sensitive drugs against all isolates. The sensitivity rate of carbepenems among uropathogens was as follow $E$ coli Imipenem (95.06\%) and Meropenem (95.06\%), for Klebsiella pneumoniae Imipenem (95.24\%), Meropenem (90.48\%), for Ps aeruginosa Imipenem (87.72\%), and for Proteus mirabilis Imipenem( $88.89 \%)$ and Meropenem (88.89\%). These antibiotic susceptibility results similar to other previously reported studies (25). In case of Gram positive uropathogens such as $S$. aureus and Enterococcus faecalis were showed high 
susceptibility frequency towards Vancomycin, Teicoplanin. These two antibiotics were reported $60-100 \%$ sensitive for both Gram positive bacteria. Other sensitive antibiotics were Tetracycline and Doxycycline which accounted for $62.50 \%$.

This study highlights the need for the development of few generic drugs; otherwise the resistant to flouroquinolones, the cheapest of the drugs that remains highly efficient will increase rapidly in the future.

The study concluded that ESBL producing Gram negative bacilli such as $E$ coli, $K$. pneumonia, and Proteus mirabilis are the main uropathogen responsible for causing UTIs. Of concern is that the resistance rate of these pathogens against commonly used (flouroquinolones and cephalosporins) antibiotics has increased. Nitrofurantoin should be recommended for the first line empirical oral treatment of UTI and also Carbepenems (Imipenem and Meropenem) and Amikacin showed a good antimicrobial activity against ESBL producing isolates. Vancomycin and Teicoplanin are effective in treating UTI due to Gram positive cocci. However, the mechanism on bacterial resistance is complex and diverse, and the phenomenon of multidrug-resistance (MDR) bacteria has become a global burden. Susceptibility testing is a valuable tool to help in the selection of antibiotic treatment.

\section{References}

1.Bradford PA (2001) ESBL in $21^{\text {st }}$ century: characterization, epidemiology and detection of this important resistant treat. Clin microbial Rev, 14: 933-51

2.Bonadio $\mathrm{M}$ et al. Micribiological and clinical aspects of urinary tract infections, Eur J Urol. 2001; 40:439-45

3.Grude $\mathrm{N}$ et al. Urinary tract infections in Norway: bacterial etiology and susceptibility, a retrospective study of clinical isolates, Clin Micribiol Infect.2001; 7:543-47.

4.Public health England, Extended Spectrum Beta-lactamases guidance 2013.

5.Nordmann P., and Guibert M. (1998), extended-spectrum beta-lactamases in Pseudomanas aeruginosa. J Antimicrob Chemother,42: 128-131.

6.Du Bios, S.K., Marriott, M.S., and Amyes, S.G. (1995). TEM and SHV derived extended-spectrum beta-lactamases: relationship between section, structure and function. J. Antimicrob. Chemother, $35,7-22$.

7.Winokur P.L., D.L. DeSalvo, Hoffmann et al. (2000). Animal and human multidrug-resistant, cephalosporinresistant Salmonella isolates expressing a plasmid mediated CMY-2 AmpC beta-lactamases. Antimicrob agents chemother, 44, 2777-2783.

8.J.G. et al. "tests for identification of bacteria," in Mackie and Mc Artney Practical Medical Microbiology, Eds.,p. 433, Churchill Livingstone, London,UK, 1996.

9.Clinical and Laboratory Standards Institute (CLSI), "Document M100-S17. Performance standards for antimicrobial susceptibility testing," CLSI, 940 West Valley Road, Suite 1400, Wayne, Pennsylvania 19087-1898,USA, 2007.

10.Abu Shaqra Q (2000) Occurrence and antibiotic sensitivity of Enterobacteriaceae isolated from a group of Jordanian patients with community acquired urinary tract infections. Cytobios 101:15-21.

11.A. J. Schaeffer, N. Rajan, Q. Cao et al., "Host pathogenesis in urinary tract infections," International Journal of Antimicrobial Agents, vol. 17, no. 4, pp. 245-251, 2001.

12. Gatermann SG. Bacterial infections of the urinary tract. In; Borriello P,Murray PR, 
FunkeG. Topley \& Wilson's microbiology \& microbial infections, $10^{\text {th }} \quad$ ed.vol.III.London: Hodder Arnold Publisher;2007

13.Karlowsky JA, Jones ME, Thornsberry C, Critchley I , Kelly LJ, Sahm DF. Prevalence of antimicrobial resistance among urinary tract pathogens isolated from female outpatients across the USA in 1999. Int J Antimicrob Agents 2001.

14.Hooper DC. Emerging mechanisms of fluoroquinolone resistance. Emerg infect Dis 2001

15.Canbaz S, Peksen Y, Tevfik SA Leblebicioglu H, sunbul M. Antibiotic Agents 2002.

16.Lu-Dong Qiao, Shan Chen et al. BMJ Open 2013;3:e004152 doi:10.1136/ bmjopen-2013-004152.

17.Olafsson $M$ et al.(2000) Urinary tract infections, antibiotic resistance and sales of antimicrobial drugs-an observational study of uncomplicated urinary tract infections in Icelandic women. Scand J Prim Health Care 18:35-8.

18.Mukherjee M, Basu S, Mukherjee SK, et al. Multidrug-resistance and extended spectrum beta-lactamase production in uropathogenic E. Coli which were isolated from hospitalized patients in Kolkata, India. J Clin Diagn Res 2013;7:449-53.

19. Odongo CO, Anywar DA, Luryamamoi K, et al. Antibiograms from communityacquired uropathogens in Gulu, northern Uganda - a cross-sectional study. BMC Infect Dis 2013;13:193.

20.Guangying Y, Xueyin Z, Anrong L. Development of a novel test identifying extended-spectrum beta lactamase producing strains relative to resistant plasmids. Chin J Microecol 2002;

21.Lei G, Yonghong X. 2006-2007 Mohnarin report:Bacterial distribution and resistance in urinary tract infections. Chinese J Antibiotics, 2008.

22. Hua Y, Hua L, Yingjun Y. Distribution of pathogens and resistance detection in urinary infection. Chinese J Nosocomiol 2003.

23.Schaeffer AJ, Rajan N, Cao Q, Anderson BE, Pruden DL, Sensibar J, Duncan JL (2001) Host pathogenesis in urinary tract infections. Int J Antimicrob Agents 17:245-51.

24.Shigemura K, Tanaka K, Okada H, Nakano Y, Kinoshita S, Gotoh A, Arakawa S, Fujisawa M (2005) Pathogen occurrence and antimicrobial susceptibility of urinary tract infection cases during a 20 -year period (19832002) at a single institution in Japan. Jpn J infects Dis 58:303-8.

25 Alipourfard and N. Y. Nili, "Antibiogram of Extended Spectrum Beta-lactamase (ESBL) producing Escherichia coli and Klebsiella pneumoniae isolated from Hospital Samples," Bangladesh Journal of Medical Microbiologyno, vol. 4, no. 4, pp. 32-36, 2010.

\section{How to cite this article:}

Arti Agrawal, Namita Srivastava, Vikas Kumar, Neha Bhati, Rashmi and Ankur Goyal. 2017. Extended-Spectrum Beta - Lactamases Producing Micro Organisms Isolated from UTI Patients: an Alarm. Int.J.Curr.Microbiol.App.Sci. 6(10): 5071-5078. doi: https://doi.org/10.20546/ijcmas.2017.610.481 